PROCEEDINGS OF THE

AMERICAN MATHEMATICAL SOCIETY

Volume 130, Number 4, Pages 1169-1176

S 0002-9939(01)06153-6

Article electronically published on September 14, 2001

\title{
ON LEGENDRIAN KNOTS AND POLYNOMIAL INVARIANTS
}

\author{
EMMANUEL FERRAND
}

(Communicated by Ronald A. Fintushel)

\begin{abstract}
It is proved in this note that the analogues of the Bennequin inequality which provide an upper bound for the Bennequin invariant of a Legendrian knot in the standard contact three dimensional space in terms of the least degree in the framing variable of the HOMFLY and the Kauffman polynomials are not sharp. Furthermore, the relationships between these restrictions on the range of the Bennequin invariant are investigated, which leads to a new simple proof of the inequality involving the Kauffman polynomial.
\end{abstract}

\section{INTRODUCTION}

The standard contact three dimensional space is $\mathbb{R}^{3}$ with coordinates $u, p, q$ endowed with the plane field induced by the the contact form $\alpha=d u-p d q$ and the orientation induced by the volume form $d u \wedge d p \wedge d q$. A smooth knot embedded in $\mathbb{R}^{3}$ is called Legendrian if it is everywhere tangent to this plane field. A Legendrian knot is completely determined by its projection to the plane $(p, q)$, the other coordinate $u$ being the integral of the form $p d q$ along the knot projection. Any smooth plane curve unambiguously defines a Legendrian immersion in $\mathbb{R}^{3}$ provided that the integral of $p d q$ vanishes along this curve (so that the Legendrian "lift" is closed). Any Legendrian knot is horizontal (i.e. the tangent vector is never vertical) with respect to this projection. Hence a contact isotopy (a one parameter family of Legendrian knots) is a particular case of what is classically called a regular isotopy (the first Reidemeister move is forbidden on knot projections). Two horizontal oriented knots are regular isotopic if and only if they are isotopic and have the same writhe $w$ (self-linking number with respect to the vertical framing) and the same Whitney index $r$ (degree of the Gauss map of the knot projection). Let $l$ be some Legendrian knot. By definition, its Bennequin invariant is $t b(l)=-w(l)$ and its Maslov invariant is $\mu(l)=r(l)$.

The following restrictions are known (see below for a list of the authors of these results):

Theorem. a) $t b(l)+|\mu(l)| \leq 2 . g_{4}(l)-1$.

b) $t b(l)+|\mu(l)| \leq e_{P}(l)$.

c) $t b(l) \leq e_{Y}(l)$.

Received by the editors July 11, 2000 and, in revised form, October 24, 2000.

1991 Mathematics Subject Classification. Primary 53C15, 57M25.

Key words and phrases. Contact topology, polynomial invariants of knots.

(C)2001 American Mathematical Society 
Here $g_{4}(l)$ denotes the slice genus of $l, e_{P}(l)$ (resp. $e_{Y}(l)$ ) the least degree of the framing variable $a$ in the HOMFLY (resp. Kauffman) polynomial of $l$ (see below for the precise definition and normalization of these polynomials).

In this paper, the relationships between these three inequalities are investigated. A new proof of c) is given. It is shown that in spite of the misleading evidence provided by the knot tables, there is no inequality like $e_{P} \leq 2 g_{4}-1$, from which it would follow that b) implies a). We provide examples showing that inequality $e_{Y} \leq e_{P}$ is false, again in spite of what could be expected from the tables. As a consequence, none of the three inequalities above is sharp.

\section{KNOWN RESULTS}

\subsection{Inequalities.}

- Consider a braid $\sigma$ with $n(\sigma)$ strands and whose exponent sum is $c(\sigma)$. Denote by $\hat{\sigma}$ the closure of this braid and let $I$ be some knot invariant that does not detect the orientation of knots. It follows from [Be] (theorem 8, proposition 6 , and paragraph 24) that if the inequality $-c(\sigma)-n(\sigma) \leq I(\hat{\sigma})$ holds for any braid $\sigma$, then, for any Legendrian knot $l$ having topological knot type $K$, $t b(l)+|\mu(l)| \leq I(K)$.

- Bennequin [Be] (theorem 3) proved that $|c(\sigma)|-n(\sigma) \leq 2 g_{3}(\hat{\sigma})-1$, where $g_{3}$ denotes the genus. Hence, by the previous discussion, $t b(l)+|\mu(l)| \leq 2 g_{3}(l)-1$ ([Be], theorem 11).

- In [Ru5], Lee Rudolph proved that, for any braid $\sigma,|c(\sigma)|-n(\sigma) \leq 2 g_{4}(\hat{\sigma})-1$, and hence inequality a) follows.

- In [Mo] and [FW], Morton and Franks-Williams proved, using "elementary" combinatorial means, that $-c(\sigma)-n(\sigma) \leq e_{P}(\hat{\sigma})$. As observed in FT, inequality b) follows from this and the preceding discussion.

- An inequality similar to inequality c) with $e_{Y}$ replaced by the least degree of the framing variable in the Kauffman polynomial reduced modulo 2 was obtained by Fuchs and Tabachnikov [FT].

- Inequality c) was proved by Tabachnikov $[\mathrm{Ta}]$ using Turaev's state model for the Kauffman polynomial.

- Using another approach, Chmutov, Goryunov and Murakami [CGM] proved inequality b) and Chmutov and Goryunov CG proved inequality c). In CGM, CG, Ta, inequalities are stated in the more general context of the contact manifold $S T^{*} \mathbb{R}^{2}$. Note also that an analogue of inequality b) for transversal knots in $S T^{*} \mathbb{R}^{2}$ is proved independently in [GH] and in [Ta].

- All the results mentioned in this section have a counterpart in Lee Rudolph's theory of quasi-positive links. See Ru1, Ru2, Ru3, Ru4, Ru5, Ru6 for analogues of inequalities a), b) and c).

- Tanaka [Tan] has shown that inequality c) is a consequence of [Yo, lemma 1, which itself relies on Turaev's state model for the Kauffman polynomial.

\footnotetext{
${ }^{1}$ The sign difference with $[\mathrm{Be}]$ is due to the fact that we use a different contact form and a different orientation.

${ }^{2}$ We follow [Kau] for the normalization of the Homfly polynomial. This explains the difference with the original inequality of $[\mathrm{MO}$, where different variables and a different normalization are assumed.
} 
2.2. Non-Sharpness. Below, when it is stated that an inequality of the form

(contact isotopy invariant) $\leq$ (topological invariant)

is not sharp, this means that there exists some topological knot types $K$ such that the supremum of all the values of this contact isotopy invariant computed on all Legendrian representatives of $K$ is less than the value of the topological invariant computed on $K$.

- Using topological methods, Y. Kanda $\mathrm{Ka}$ has computed the maximal Bennequin number realizable by a Legendrian representative of some Pretzel knots, showing that the bound $t b(l) \leq 2 . g_{3}(l)-1$ is not sharp for these knot types. The same result follows from Rudolph's $\mathrm{Ru} 3$, modulo the identification in [Ru6] of $T B(K)=\max \{t b(l) ; l$ has topological type $K\}$ with the invariant $q(K)$ (which was defined, using another symbol, in [Ru1]). See also Ru4.

- J. Epstein $\mathrm{Ep}$ and L. Ng $[\mathrm{Ng}$ have conjectured non-sharpness of inequality c) for the knot $8_{19}$. This was proved by J. Etnyre and K. Honda [EH], as a byproduct of their classification of Legendrian torus knots.

- Sharpness has been established for some specific knot types (see, for example, Tan, $[\mathrm{Ep},(\mathrm{Ng})$.

- Inequality a) is not optimal already at the level of concordance classes (see $[\mathrm{Fe}]$.

\section{KNOT POLYNOMials}

Here, the precise definition of the topological invariants $e_{P}$ and $e_{Y}$ is given. We follow the normalization of [Kau], pp. 215-222.

To any regular oriented knot projection we associate $R$, a Laurent polynomial in the variables $z$ and $a$ defined by the following skein relations:

$$
\begin{aligned}
& R(\bigcirc)=\frac{a-a^{-1}}{z},
\end{aligned}
$$

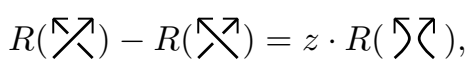

$$
\begin{aligned}
& R(\mathcal{\bigcirc})=a \cdot R(\supset),
\end{aligned}
$$

where $R$ is a regular isotopy invariant and the HOMFLY polynomial $P(z, a)=$ $a^{-w} R(z, a)$ (where $w=\sharp \nwarrow \nearrow-\sharp \nearrow^{\nearrow}$ ) is a knot invariant. The least exponent of the variable $a$ in $P$ is denoted by $e_{P}$. It is known that $P$ is independent of the orientation, and that $e_{P}+1$ is an additive knot invariant with respect to connected sum.

Example. $P(\widehat{\widehat{ح}})=a^{-3}\left(\frac{a-a^{-1}}{z}\right)\left(2 a-a^{-1}+a z^{2}\right)$; hence $e_{P}(\widehat{\widehat{ح}})=-5$.

To any regular knot projection we associate $D$, a Laurent polynomial of the variables $z$ and $a$ defined by the following skein relations:

$$
\begin{gathered}
D(\bigcirc)=\frac{a-a^{-1}}{z}+1, \\
D(\text { 久 }-D(\searrow)=z \cdot(D()()-D(\asymp)), \\
D(\text { ○) }=a \cdot D(\supset),
\end{gathered}
$$


where $D$ is a regular isotopy invariant and the Kauffman polynomial $Y(z, a)=$ $a^{-w} D(z, a)$ is a knot invariant. The least exponent of the variable $a$ in $Y$ is denoted by $e_{Y}$. It is known that $e_{Y}+1$ is an additive knot invariant with respect to connected sum.

Example. $Y(\widehat{Ð})=a^{-3}\left(1+\frac{a-a^{-1}}{z}\right)\left(2 a-a^{-1}+z-a^{-2} z+a z^{2}-a^{-1} z^{2}\right)$; hence $e_{Y}(\widehat{ح})=-6$.

\section{The JAEGER FORMUlA}

This formula (see $\mathrm{Kau}$, pp. 219-222) shows that the Kauffman polynomial of some knot can be computed from the HOMFLY polynomials of the knots obtained by "splicing" a regular projection of this knot at some crossings.

Consider a link diagram $K$. A state $\sigma$ is the following data: A link $K_{\sigma}$, obtained from $K$ by splicing some of the crossings $(\mathcal{K}$ is modified into $\searrow$ or $)($, or is left unchanged), and an orientation of $K_{\sigma}$. A state $\sigma$ being given, a local weight is associated with each crossing $x$ of $K$. If $x$ does not belong to the spliced crossings, then the local weight of $x$ is one. Consider now an $x$ that belongs to the spliced crossings and suppose that $x=\mathrm{K}$ before splicing. There are 8 possible local pictures. If $x$ is spliced to $\backslash$, then the weight of $x$ is $\left(t-t^{-1}\right)$. If $x$ is spliced to $\preceq$, then the weight of $x$ is $-\left(t-t^{-1}\right)$. The weight of $x$ vanishes in all remaining possibilities. The weight of $\sigma$, denoted by $[K, \sigma]$, is the product of all these local weights. Denote by $r_{\sigma}$ the degree of the Gauss map (Whitney index) of the oriented plane curve underlying the knot diagram $K_{\sigma}$.

Theorem (Jaeger). $D(K)\left(t-t^{-1}, a^{2} t^{-1}\right)=\sum_{\sigma}\left(t a^{-1}\right)^{r_{\sigma}}[K, \sigma] R\left(K_{\sigma}\right)\left(t-t^{-1}, a\right)$.

\section{The Legendrian version of the Jaeger formula}

It is a reformulation of the formula above in terms of the projection of Legendrian knots in the plane $(q, u)$, called fronts. These are not regular projections. A generic front has transverse self-intersections $X$ and semi-cubic cusps like $\langle$ or $\rangle$. It has no vertical tangent. A typical front is $\lesssim$. A generic front completely determines the Legendrian knot which lies above, hence in the sequel a Legendrian $k n o t l$ is identified with its front when there is no ambiguity.

To the (generic) front of some Legendrian knot $l$, a generic knot diagram, called the morsification of the front, is associated by the following rule: Each crossing $X$ is modified to $\mathcal{~}$. Each cusp pointing leftward $<$ is modified to $C$. Each cusp pointing rightward $>$ is modified to $\bigcirc$. For example, the morsification of $\infty$ is $\bigcirc$.

Claims. The morsification of the front of $l$ is such that:

- The corresponding knot has the topological type of $l$.

- The Whitney index of the morsification is $r=-\mu(l)$.

- The writhe of the morsification is $w=-t b(l)$.

- The regular isotopy type of the morsification is invariant under Legendrian isotopy.

$R$ and $D$ are defined for regular knot diagrams. Observe that $D$ is defined for unoriented diagrams, and that inverting the orientation leaves $R, t b$ and $w$ invariant (but changes the sign of $\mu$ ). In the sequel, $R(l)$ (resp. $D(l)$ ) denotes the 
polynomial computed by applying skein relations to the morsification of the front of $l$. However this is the same as the polynomial computed applying skein relations to the (generically regular) projection of $l$ in the plane $(p, q)$.

A state of the front of $l$ consists of the following data: A front $l_{\sigma}$ obtained from the one of $l$ by splicing some crossings ( $X$ can be modified to $\asymp$, or $><$, or left unchanged) and the choice of an orientation of the resulting $l_{\sigma}$. A state $\sigma$ of $l$ being given, to each crossing $x=\chi$ of $l$, a local weight is associated. If $x$ is left unspliced, then its weight is one. Suppose now that $x$ belongs to the spliced crossings. There are 8 possible local pictures. If $x$ is spliced to

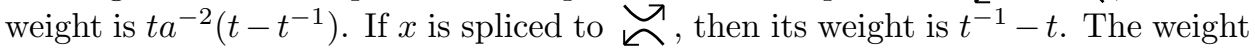
of $x$ vanishes in all remaining possibilities. The weight of $\sigma$, denoted by $[l, \sigma]$, is the product of all the local weights. Denote by $\sharp \nearrow$ (resp. $\sharp \searrow$ ) the number of cusps of $l_{\sigma}$ which point leftward (resp. rightward) and which are oriented upward (resp. downward).

Using this language, the Jaeger formula translates to (see the proof below)

$$
D(l)\left(t-t^{-1}, a^{2} t^{-1}\right)=\sum_{\sigma}\left(a t^{-1}\right)^{\sharp} \succ_{+\sharp} \succ[l, \sigma] R\left(l_{\sigma}\right)\left(t-t^{-1}, a\right) .
$$

Example 1. $R(\prec)=R(\bigodot)=\frac{a^{2}-1}{z}$ and $D(\prec)=D(\bigodot)=a+\frac{a^{2}-1}{z}$. There are two states for $\prec$ (the two possible orientations). Hence the right hand side of $(L J)$ is

$$
\left(t a^{-1}\right)^{0} \frac{a^{2}-1}{\left(t-t^{-1}\right)}+\left(a t^{-1}\right)^{2} \frac{a^{2}-1}{\left(t-t^{-1}\right)} .
$$

This is equal to $D\left(t-t^{-1}, a^{2} t^{-1}\right)=\left(a^{2} t^{-1}\right)\left(1+\frac{a^{2} t^{-1}-a^{-2} t}{t-t^{-1}}\right)$, as expected.

Example 2. $R(\diamond)=R(\bigodot)=\frac{a^{3}-a}{z} \cdot D(\diamond)=D(\bigodot)=$ $a^{2}+\frac{a^{3}-a}{z}$. There are 4 states whose weights do not vanish: $\circlearrowright<$, and $\prec$. The right-hand side of $(L J)$ is

$$
\begin{aligned}
\left(a t^{-1}\right) \frac{a^{3}-a}{t-t^{-1}} & +\left(a t^{-1}\right) \frac{a^{3}-a}{t-t^{-1}}+\left(a t^{-1}\right)^{4}\left(t a^{-2}\right)\left(t-t^{-1}\right)\left(\frac{a^{2}-1}{t-t^{-1}}\right)^{2} \\
& +\left(a t^{-1}\right)^{2}\left(t^{-1}-t\right) \frac{a^{2}-1}{t-t^{-1}} .
\end{aligned}
$$

This is equal to $D\left(t-t^{-1}, a^{2} t^{-1}\right)=\left(a^{2} t^{-1}\right)^{2}\left(1+\frac{a^{2} t^{-1}-a^{-2} t}{t-t^{-1}}\right)$, as expected.

Proof of $(L J)$. Consider some Legendrian knot $l$, and the knot diagram $K$ obtained by rounding all the cusps of the front of $l(\langle$ becomes $($ and $\succ$ becomes $)$ ). Denote by $\nu$ half the number of cusps of $l$. By the axioms for $D$,

$$
D(l)\left(t-t^{-1}, a^{2} t^{-1}\right)=\left(a^{2} t^{-1}\right)^{\nu} D(K)\left(t-t^{-1}, a^{2} t^{-1}\right) .
$$

There is a one-to-one correspondence between the states of $l$ and the states of $K$. Writing the Jaeger formula for $K$ in terms of $l$ will give $(L J)$ : Consider some state $\sigma$ of $l$. Denote by $\nu_{\sigma}$ half the number of cusps of $l_{\sigma}$ and by $V$ (resp. by $H$ ) the number of crossings of $l$ (or of $K$ ) that are spliced vertically (resp. horizontally) in $\sigma$. The following relations hold: $R\left(K_{\sigma}\right)=a^{-\nu_{\sigma}} R\left(l_{\sigma}\right),[K, \sigma]=(-1)^{H}\left(t-t^{-1}\right)^{V+H}$, 
$\nu=\nu_{\sigma}-V$, and $\nu_{\sigma}-r\left(K_{\sigma}\right)=\sharp \prec+\sharp \succsim$. Plug this into the expression of $D(l)$ above:

$$
\begin{aligned}
D & (l)\left(t-t^{-1}, a^{2} t^{-1}\right) \\
= & \sum_{\sigma}\left(a t^{-1}\right)^{\sharp} \succ_{+\sharp}
\end{aligned}
$$

This is $(L J)$.

\section{INEQUALITY c) FOLLOWS FROM INEQUALITY b)}

Since $t b=-w$, inequality $\mathrm{b}$ ) is equivalent to the fact that there is no negative power of $a$ occurring in $a^{-|\mu|} R(l)$, i.e., it is a genuine polynomial in a. Similarly, we want to prove that $D(l)$ is a genuine polynomial in $a$. This is a consequence of the following lemma.

Lemma. The contribution of each state in the right-hand side of $(L J)$ is a genuine polynomial in a.

Proof. Consider a state $\sigma$ of $l$. Denote by $V$ the number of crossings that are spliced to $\precsim$, and by $H$ the number of crossings that are spliced to $\nwarrow$. The contribution of $\sigma$ is

$$
\left(a t^{-1}\right)^{\sharp}{ }_{+\sharp} \succ\left(t a^{-2}\right)^{V}(-1)^{H}\left(t-t^{-1}\right)^{V+H} R\left(l_{\sigma}\right)\left(t-t^{-1}, a\right) .
$$

The least exponent of $a$ in $R\left(l_{\sigma}\right)\left(t-t^{-1}, a\right)$ is not less than $\left|\mu\left(l_{\sigma}\right)\right|$, by inequality b). Denote by $E$ the least exponent of $a$ in the contribution of $\sigma . E \geq \sharp\langle+\downarrow$

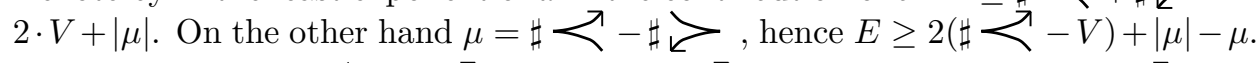
Since splicing $\chi$ to $\gtrsim \succ$ creates one $\nearrow, V$ is not bigger than $\sharp \nearrow^{2}$, and hence $E \geq 0$.

Remark about this proof. As explained in [FT], inequality b) has a "simple" and natural proof by $[\mathrm{Be}$ and $[\mathrm{Mo}]$ or $[\mathrm{FW}]$, much simpler than the known proofs of a) for instance. Since the Jaeger formula is also proved ( $\mathrm{Kau}$ ) by "elementary" means (like checking its invariance under the Reidemeister moves), this proof of c) is, in my opinion, simple and natural. I find it remarkable that the Jaeger formula fits so well between b) and c).

Remark. Like a), b) follows from a more general inequality about transverse knots (see $[\mathrm{Be}, \mathrm{Ta}, \mathrm{GH}])$. The proof above, which lacks of a natural transverse counterpart, seems to indicate that c) is an inequality about Legendrian knots only.

\section{Relationship BETWEen $g_{4}$ And $e_{P}$}

It is proved here that inequality a) can be stronger than inequality b).

Proposition. The difference between $e_{P}$ and $2 \cdot g_{4}-1$ can be arbitrarily negative or positive.

Corollary. Inequality b) is not sharp, i.e.

$$
\max \{\operatorname{tb}(l)+|\mu(l)| ; \quad l \text { has topological type } K\}<e_{P}(K)
$$

for some knot types $K$. 
Remark. $2 \cdot g_{4}-1<e_{P}$ seems much more difficult to realize than the converse: The tables indicate no contradiction to $e_{P}(K) \leq 2 \cdot g_{4}(K)-1$ for the 84 first knots (which arise from diagrams with less than 10 crossings).

Question. This leaves the question of Morton $\mathrm{Mo}$ open: Is it true that $e_{P}(K) \leq$ $2 \cdot g_{3}(K)-1$ ? (Recall that $g_{3}$ denotes the genus.) This inequality is true for alternating knots and for positive knots, as proved in $[\mathrm{Cr}$, and for knots with braid index less than $4[\mathrm{DM}]$. It was checked by Alexander Stoimenow for all knots which admit a diagram with less than 17 crossings.

Proof of the Proposition. Consider some knot $K$ such that $e_{P}+1$ is negative. For

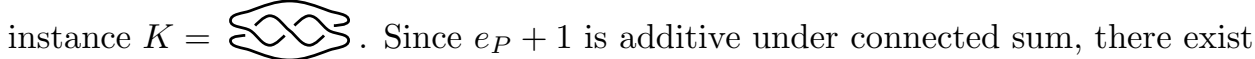
knots with arbitrarily negative $e_{P}$. On the other hand, $g_{4}$ is never negative. Hence $e_{p}-\left(2 \cdot g_{4}-1\right)$ can be made arbitrarily negative.

Let $K$ be the closure of the braid $\sigma=$ This knot admits a projection with ten crossings. Changing the first of $\sigma$ to $=$, one gets a braid whose closure is the trivial knot. Hence $g_{4}(K) \leq 1$ (it is in fact 1 ). Computation shows that $e_{P}(K)=3$ (hence $K$ is an example for which b) is not sharp). Denote by $K^{\sharp d}$ the connected sum of $k$ copies of $K . g_{4}\left(K^{\sharp d}\right) \leq d$, and $e_{P}\left(K^{\sharp d}\right)=d(3+1)-1$. Hence $e_{P}-\left(2 \cdot g_{4}-1\right)$ can be made arbitrarily positive.

\section{Relationships Between inequalities b) And c)}

By section [ 6 inequality b) implies inequality c). However, inequalities b) and c) are independent in the following sense: b) implies that $t b(l) \leq e_{P}(l)$. Looking in the tables seems to indicate that $e_{Y} \leq e_{P}$, and hence that $t b(l) \leq e_{P}(l)$ is weaker than $t b(l) \leq e_{Y}(l)$ (inequality $\left.\mathrm{c}\right)$ ). This is however not true. Among all the prime knots which admit a diagram with less than 15 crossings (there are grosso-modo 60.000 of them), there are 22 knots verifying $e_{P}<e_{Y}$. One of the two examples with 12 crossings is the closure of the following braid:

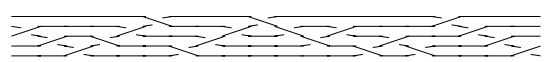

Corollary. Inequality c) is not sharp.

Question. Is it true that $e_{Y} \leq e_{P}$ for alternating knots (none of the 13 examples cited above is alternating)?

\section{ACKNOWLEDGEMENTS}

I learned the Jaeger formula from Christan Blanchet's lectures at the summer school on finite type invariants of knots and three manifolds, Grenoble, 1999. All computations of knot polynomials used throughout this paper are due to Jim Hoste and Morwen Thistlethwaite, via their Knotscape program [HT. Further computer aided example search was programmed by Xavier Dousson and Alexander Stoimenow. I thank them all very much.

\section{REFERENCES}

[AG] Arnold, V., Givental, A. : Symplectic geometry, Encyclopedia of Mathematical Science vol. 4, Springer-Verlag.

[Be] D. Bennequin : Entrelacements et équation de Pfaff. Astérisque 107-108 (1983), 83-161. MR 86e:58070 
[CG] Chmutov, S., Goryunov, V. : Polynomial invariants of Legendrian links and wave fronts, KNOTS '96 (Tokyo), World Sci. Publishing, River Edge, NJ, 1997, 239-256. MR 99k:57010

[CGM] Chmutov, S., Goryunov, V., Murakami, H. : Regular Legendrian knots and the HOMFLY polynomial of immersed plane curves, Math. Ann. 317 (2000), 389-413. CMP 2000:16

[Cr] Cromwell, P.R. : Homogeneous links J. London Math. Soc. (2) 39 (1989), no. 3, 535-552. MR 90f:57001

[DM] Dasbach, O., Mangum, B.S. : On McMullen's and other inequalities for the Thurston norm of link complements, preprint, 1999.

[EH] Etnyre, J. B., Honda, K. : Knots and contact geometry, arXiv:math.GT/006112.

[Ep] Epstein, J. : On the Invariants and Isotopies of Legendrian and Transverse Knots, dissertation, U. C. Davis, (1997).

[Fe] Ferrand, E. : On Legendre cobordisms Amer. Math. Soc. Transl. (2) Vol. 190, (1999), 23-35. CMP 2000:08

[FW] Franks, J., Williams, R. F. : Braids and the Jones polynomial Trans. Amer. Math. Soc. 303 (1987), no. 1, 97-108. MR 88k:57006

[GH] Goryunov, V. V., Hill, J. W. : A Bennequin number estimate for transverse knots, Singularity theory (Liverpool, 1996), London Math. Soc. Lecture Note Ser., 263, (1999), 265-280. MR 2000f:57006

[FT] Fuchs, D., Tabachnikov, S. : Invariants of Legendrian and transverse knots in the standard contact space, Topology 36 (1997), no. 5, 1025-1053. MR 99a:57006

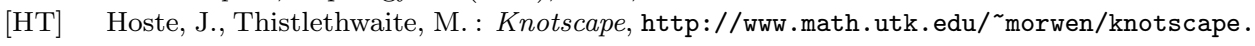
html

[Ka] Kanda, Y. : On the Thurston-Bennequin invariant of Legendrian knots and non exacteness of the Bennequin inequality, Invent. Math. 133 (1998), no. 2, 227-242. MR 99g:57030

[Kau] Kauffman, L. : Knots and Physics, World Scientific, 1991. MR 93b:57010

[Mo] Morton, H. : Seifert circles and knot polynomials, Math. Proc. Cambridge Philos. Soc. 99 (1986), no. 1, 107-109. MR 87c:57006

[Ng] Ng, L. : Maximal Thurston-Bennequin number of two-bridge knots, preprint.

[Ru1] Rudolph, L. : Construction of quasipositive knots and links, II, Contemp. Math. 35, 485-491. MR 86f:57005

[Ru2] Rudolph, L. : A congruence between link polynomials, Math. Proc. Camb. Phil. Soc. (1990) 107 319-327. MR 90k:57010

[Ru3] Rudolph, L. : Quasi-positive annuli (construction of quasipositive knots and links, IV), J. Knot Th. Ramifications (1992) 1, 4, 461-466. MR 94c:57017

[Ru4] Rudolph, L.: Totally tangential links of intersection of complex curves with round spheres, Topology'90 (eds. Apanasov et al.), DeGryuter, 1992, 343-349. MR 94d:57027

[Ru5] Rudolph, L. : Quasipositivity as an obstruction to sliceness, Bull. A.M.S. (1993) 29, 1, 51-59. MR 94d:57028

[Ru6] Rudolph, L. : An obstruction to sliceness via contact geometry and "classical" gauge theory, Invent. Math. 119 (1995), 155-163. MR 95k:57013

[Ta] Tabachnikov, S. : Estimates for the Bennequin number of Legendrian links from state models for knot polynomials, Math. Res. Lett. 4 (1997), no. 1, 143-156. MR 98k:57023

[Tan] Tanaka, T. : Maximal Bennequin numbers and Kauffman polynomials of positive links, Proc. Amer. Math. Soc. 127 (1999), no. 11, 3427-3432. MR 2000b:57014]

[Yo] Yokota, Y. : Polynomial invariants of positive links, Topology 31 (1992), no. 4, 805-811. MR 93k:57028

Institut Fourier, BP 74, 38402 St Martin d'Hères Cedex, France

E-mail address: emmanuel.ferrand@ujf-grenoble.fr 